\title{
High-resolution imaging of (100) kyanite surfaces using friction force microscopy in water
}

\author{
Carlos Pimentel ${ }^{a, b}$, Enrico Gnecco ${ }^{c}$, C a r l o s M . P i n a ${ }^{a, b, *}$ \\ a Departamento de Cristalografía y Mineralogía, Universidad Complutense de Madrid, Madrid E-28040, Spain \\ b Instituto de Geociencias (UCM-CSIC), c/José Antonio Novais, Madrid E-28040, Spain \\ ${ }^{c}$ Instituto Madrileño de Estudios Avanzados en Nanociencia, IMDEA Nanociencia, Campus Universitario de Cantoblanco, Madrid E-28049, Spain
}

\section{A b s trac}

In this paper, we present high-resolution friction force microscopy (FFM) images of the (100) face of kyanite $\left(\mathrm{Al}_{2} \mathrm{SiO}_{5}\right)$ immersed in water. These images show an almost rectangular lattice presumably defined by the protruding oxygen of $\mathrm{AlO}_{6}$ polyhedra. Surface lattice parameters measured on two-dimensional fast Fourier transform (2D-FFT) plots of recorded high-resolution friction maps are in good agreement with lattice parame-ters calculated from the bulk mineral structure. Friction measurements performed along the [001] and [010] directions on the kyanite $(100)$ face provide similar friction coefficients $\mu \approx 0.10$, even if the sequences of $\mathrm{AlO}_{6}$ polyhedra are different along the two crystallographic directions.

Keywords: Kyanite; Friction force microscopy Mineral surfaces Nanotribology; Aqueous environment

1. Introduction

Kyanite, $\mathrm{Al}_{2} \mathrm{SiO}_{5}$, is an important metamorphic nesosilicate, which is commonly found in rocks formed in ancient and present subduction zones [1,2]. This mineral is the high-pressure polymorphous of the aluminium silicate group with formula $\mathrm{Al}_{2} \mathrm{SiO}_{5}$ (i.e., sillimanite, andalu-site and kyanite). This group of minerals has been used by petrologists from long time ago to estimate pressure and temperature metamorphic conditions on the basis of their phase diagram. However, the use of the $\mathrm{Al}_{2} \mathrm{SiO}_{5}$ phase diagram to infer metamorphic conditions is problematic due to the high metastabiliy of all three mineral structures, which per-sist for long periods of time under P-T conditions far from equilibrium [3]. The metastability of $\mathrm{Al}_{2} \mathrm{SiO}_{5}$ minerals is mainly due to the fact that transformations from one mineral to another are reconstructive and they involve breaking strong $\mathrm{Si}-\mathrm{O}$ and $\mathrm{Al}-\mathrm{O}$ bonds [4]. In the case of kyanite, the arrangement of $\mathrm{Si}-\mathrm{O}$ and $\mathrm{AI}-\mathrm{O}$ bonds seems to be not only responsible for its metastability, but also for its remarkable hardness an-isotropy. Therefore, the understanding of both the rheological properties of kyanite and its reactivity in geotectonic environments will be

improved by conducting detailed crystallochemical studies of this miner-al and, particularly, of its surfaces.

\footnotetext{
* Corresponding author at: Departamento de Cristalografía y Mineralogía, Universidad Complutense de
} Madrid, E-28040 Madrid, Spain. Tel.: +34 913944879; fax: +34

913944872 .

E-mail address: cmpina@geo.ucm.es (C.M. Pina).
The main industrial use of kyanite is in refractory industry as a raw material to produce mullite, an important refractory mineral. Mullite is a rare mineral that only appear in a few uneconomical mineral deposits in the world [5]. The industrial process to produce mullite from $\mathrm{Al}_{2} \mathrm{SiO}_{5}$ minerals is through calcination at temperatures higher than $1000{ }^{\circ} \mathrm{C}$ [6]. Kyanite has the lowest temperature decomposition of the three $\mathrm{Al}_{2} \mathrm{SiO}_{5}$ minerals, and therefore, it is the most suitable to produce mullite [6]. Kyanite is also used in abrasive and electrical indus-tries. Nowadays, new studies in kyanite applications propose other applications, such as its use to obtain metallurgical alumina [7]. Both the development and improvement of new industrial uses of kyanite also requires a better understanding of its crystallochemical surface properties.

In this work, we show first images and friction measurements of kyanite (100) surfaces recorded using a friction force microscopy (FFM) operating in contact mode in water. This technique was used in previous works by our group to obtain images with molecular resolu-tion of mineral surfaces, organic molecules grown on minerals and organic crystals [8-12]. In addition, this method allows us to obtain fric-tion coefficients between probing tips and surfaces along different crys-tallographic orientations. Both high-resolution images and friction coefficients obtained in this paper are discussed on the basis of the structural features of kyanite (100) surface. 


\section{Materials and methods}

\subsection{The kyanite sample}

Kyanite crystallises in the triclinic $P 1^{-}$space group with the following cell parameters: $a=0.71 \mathrm{~nm}, b=0.79 \mathrm{n} \mathrm{m}, c=0.56 \mathrm{~nm}, a=89.99^{\circ}, \beta=101.15^{\circ}, y=$ $105.95^{\circ}$ and $Z=4$ [13]. Kyanite structure can be described a sformedbychains of edge-sharing $\mathrm{AlO}_{6}$ and $\mathrm{SiO}_{4}$ polyhedra running along the $\mathrm{C}$ axis (Fig. 1). This orientation of the chains of $\mathrm{AlO}_{6}$ and $\mathrm{SiO}_{4}$ polyhedra can be related to the different hardness of kyanite, measured on different crystallographic planes. Hence, the hardness of kyanite in Mohs' scale is $\approx 7$ for [100] and [010] and $\approx$ $5 \mathrm{f}$ o r [ $\left.\begin{array}{lll}0 & 0 & 1\end{array}\right]$. Exfoliation of kyanite crystals along the (100) and (010) faces are per-fect and poor, respectively. Along the (001) plane no exfoliation occurs. The combination of these three faces results in typical fibrous habit of kyanite [14].

The sample used in this work was a kyanite from Brazil. The sample was confirmed to be kyanite (PDF number 11-0046) by X-ray powder diffraction conducted with a Siemens D-500 diffractometer, equipped with a Cu-Ka radiation source.

\subsection{Friction force microscopy (FFM)}

The study presented here was performed using a commercial AFM (Nanoscope Multimode IIla, Veeco Instruments), equipped with a $\sim 15 \times 15 \mu \mathrm{m}^{2}$ scanner and a closed fluid cell. Kyanite crystals were freshly cleaved along (100) faces with a razor blade and placed in the AFM fluid cell. Then, deionised water (Mili-Q, $18 \mathrm{M} \Omega \cdot \mathrm{c} \mathrm{m}$ ) was injected into the fluid cell. Both observations and data collection were carried out at room temperature operating in contact mode while recording height and lateral deflection signals. Scan areas ranged from $2 \times 2 \mu \mathrm{m}^{2}$ to $10 \times 10$ $\mathrm{nm}^{2}$, and the scan rates varied from $\sim 5 \mathrm{~Hz}$ to $\sim 61 \mathrm{~Hz}$. In all the cases, 512 lines per scan were record-ed. Supersharp tips mounted on triangular cantilevers (Bruker SNL$10 \mathrm{D}$ ) were used for acquiring high-resolution images.

Lateral deflection signals recorded with the AFM were used to map the friction forces while scanning square areas along the [010] and [001] directions on kyanite (100) faces at different loading forces. The experiments were carried on by applying increasing-decreasing cycles of normal force. The equation used to calculate lateral forces was as follows [16]:

$F_{\mathrm{L}}=\frac{3 h}{2 L} \times k_{\mathrm{T}} \times S \times V_{\mathrm{L}}$ where $h$ is the height of the tip and half of the cantilever thickness, $L$ is the length of the cantilever, $\mathrm{k}_{\mathrm{T}}$ is the torsional spring constant of the cantilever (calculated following Noy et al. [17]), $S$ is the sensitivity of the photodetector in $n m / V$ and $V_{L}$ is the difference of the averaged trace and retrace signals (in Volts) divided by 2. The normal force was estimated as follows [16]:

$F_{\mathrm{N}}=S \times k_{\mathrm{N}} \times V_{\mathrm{N}}$

where $k_{N}$ is the normal spring constant and $V_{N}$ is the set point value (in Volts). Both sharp silicon nitride (Bruker NP-10 D) and supersharp silicon nitride (Bruker SNL-10 D) tips were used for performing nanotribological measurements.

More than 100 AFM images were collected and analysed using the software developed by Nanoscope (5.30r3sr3) and Nanotec (WSxM) [18].

\section{Results and discussion}

\subsection{High-resolution FFM images of kyanite (100) surface}

When small areas $\left(10 \times 10 \mathrm{~nm}^{2}\right)$ ofkyanite $(100)$ surfacesarescanned almost parallel to the [010] direction, lattice resolved friction images are readily acquired. It is important to note that images with lattice resolution where obtained only when kyanite surfaces were fully immersed in water. This is mainly due to the fact that a homogeneous liquid environ-ment eliminates capillarity forces (i.e., the formation of meniscus be-tween the AFM tip and the surface), and the sliding process is not significantly perturbed by viscous forces [8-12]. As a result, the quality and resolution of recorded images is strongly improved.

The patterns observed in high-resolution friction images show an almost rectangular centred lattice defined by five maxima of the friction force (Fig. 2). The main directions on the (100) surface defined by the maxima are [010] and [001]. Since the angle between these directions is $89.99^{\circ}$ [13], the surface unit cell is almost rectangular.

Maxima in friction maps can be interpreted as the result of stick-slip phenomenon between the tip apex and selected atoms protruding out of the scanned surfaces (e.g., Pina et al. [8]). This is clearly visible in the images corresponding to a series of complete forward and backward scans along the surfaces (Fig. 3A, B), and, especially, in the cross sections showing saw-tooth profiles corresponding to long stick and much faster slip phases (Fig. 3C).

In order to correctly identify the surface atoms which originate the friction maxima during the scan, we referee to the kyanite (100) surface structure sketched in Fig. 1. As can be seen in this figure, a number of

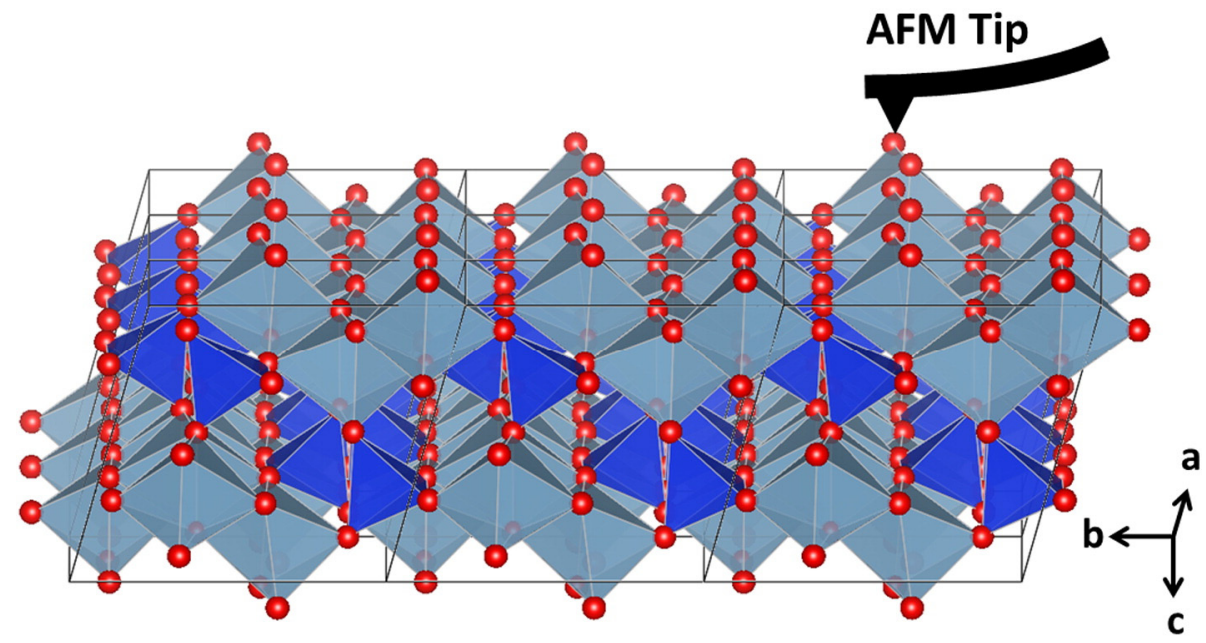

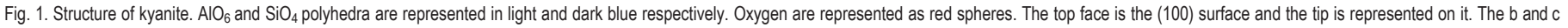
axes correspond to the [010] and [001] directions, respectively (structure projection made with Vesta [15]). (For interpretation of the references to colour in this figure, the reader is referred to the web version of this article.) 

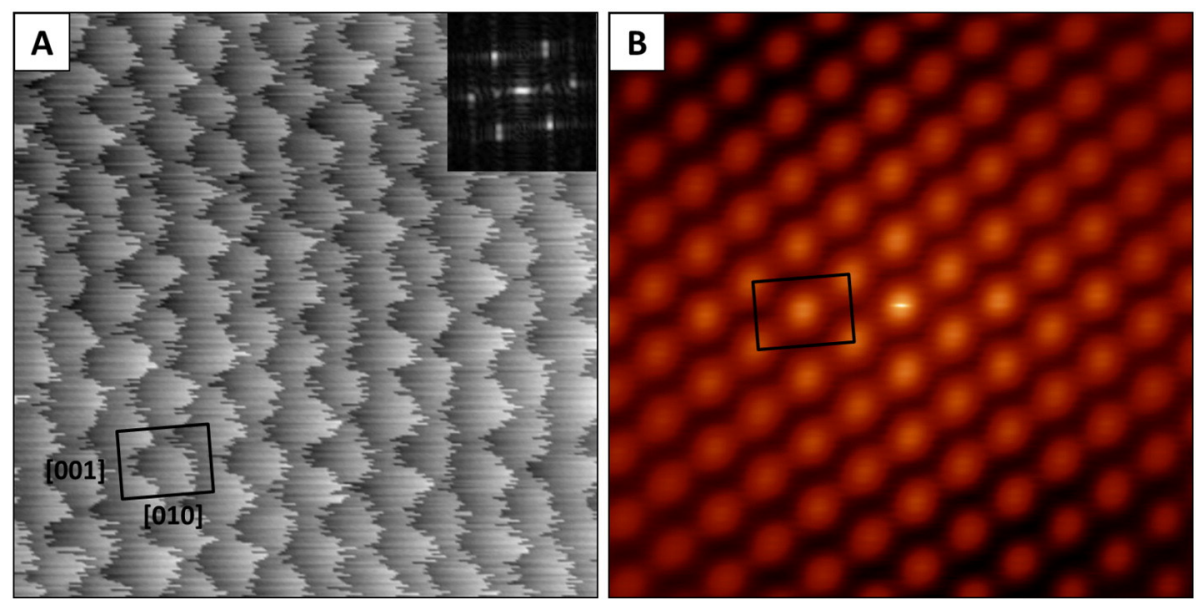

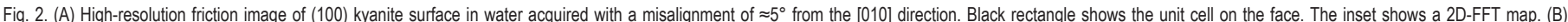

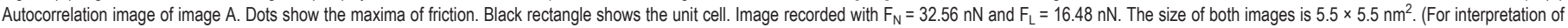
the references to colour in this figure, the reader is referred to the web version of this article.)

oxygen atoms belonging to $\mathrm{AlO}_{6}$ polyhedra protrude out of the (100) face. A similar situation was observed in Pina et al. [8], where the friction maxima detected on the (104) face of calcite and dolomite were related to oxygen atoms protruding out of the surfaces. In that case, the correspondence was supported by a numeric analysis based on the Prandtl-Tomlinson (PT) model, which predicted lateral force maps at different scan directions consistent with the zigzag arrangement of those atoms. Note that, in the case of dolomite and calcite (104) sur-faces, the shortest distance between the protruding oxygen was $0.48 \mathrm{~nm}[8]$.

In the present case, Fig. 4 shows the projection of protruding oxygen of the $\mathrm{AlO}_{6}$ polyhedra on kyanite (100) face. In this projection, two sequences of such atoms can be distinguished: (i) rows of oxygen aligned parallel to the [001] direction and (ii) oxygen running in a zigzag way along such a [001] direction. While distances between oxygen belonging to the same $\mathrm{AlO}_{6}$ polyhedron are less than $0.26 \mathrm{~nm}$, distances between oxygen belonging to contiguous polyhedra are slightly larger than $0.30 \mathrm{~nm}$. A comparison with the peaks in Fig. 2 shows that only half of the protruding oxygen atoms in the unit cell may be imaged in the friction maps. Since the distances between neigh-bour oxygen are well below those on calcite and dolomite (104), it is quite reasonable that the imaging process on kyanite $(100)$ will be more influenced by convolution with the tip apex. With this in mind, we notice that the almost centred rectangular lattice in Fig. 2 can be obtained if the tip sticks to a pair of protruding oxygen atoms coupled in two possible different schemes [respectively (i) and (ii) in Fig. 4]. The stick-slip pattern observed with an atomically sharp tip would result in a double number of peaks.

The centred rectangular lattice parameters were measured on 2D-FFT maps calculated from the high-resolution friction images. These parameters are $b_{s}=0.82 \mathrm{~nm} \pm$ 0.02 and $c_{s}=0.56 \mathrm{~nm} \pm 0.03 \mathrm{~nm}$, mea-sured along [010] and [001] directions, respectively. These values are in good agreement with the lattice parameters obtained from the kyanite bulk structure, i.e., $b_{s}=0.79 \mathrm{~nm}$ and $c_{s}=0.56 \mathrm{~nm}$ [13]. The average difference between the parameters obtained from our friction maps and those derived from kyanite structure is less than $4 \%$. This little difference indicates that kyanite (100) surfaces do not experience any significant reconstruction or relaxation phenomena in water at room temperature.

We have also imaged the kyanite (100) surface while scanning almost parallel to the [001] direction (see Fig. 5). Note that the sample was physically rotated by $90^{\circ}$ in order to perform these measurements. In this case, a characteristic distance comparable to $c_{s}\left(c_{s}{ }^{\prime}=0.52 \pm 0.03\right)$ can still be distinguished, whereas the surface lattice looks quite distorted in the [010] direction. This may be due to the (unknown) atomic arrangement at the tip apex, preventing a commensurate
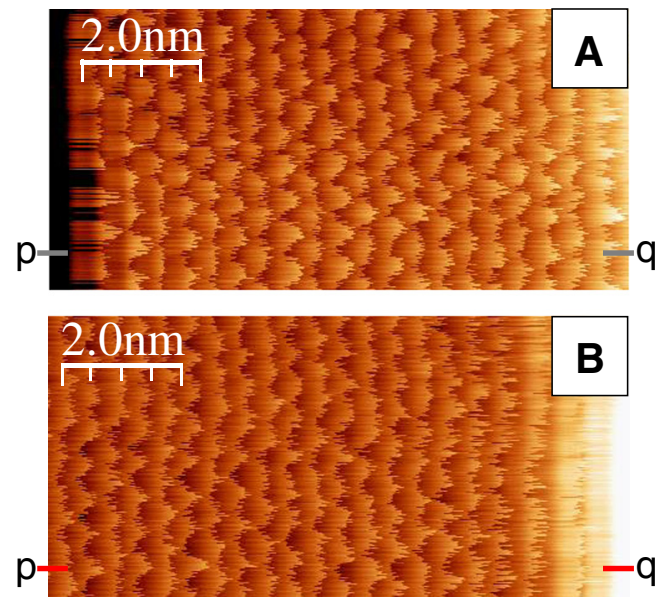

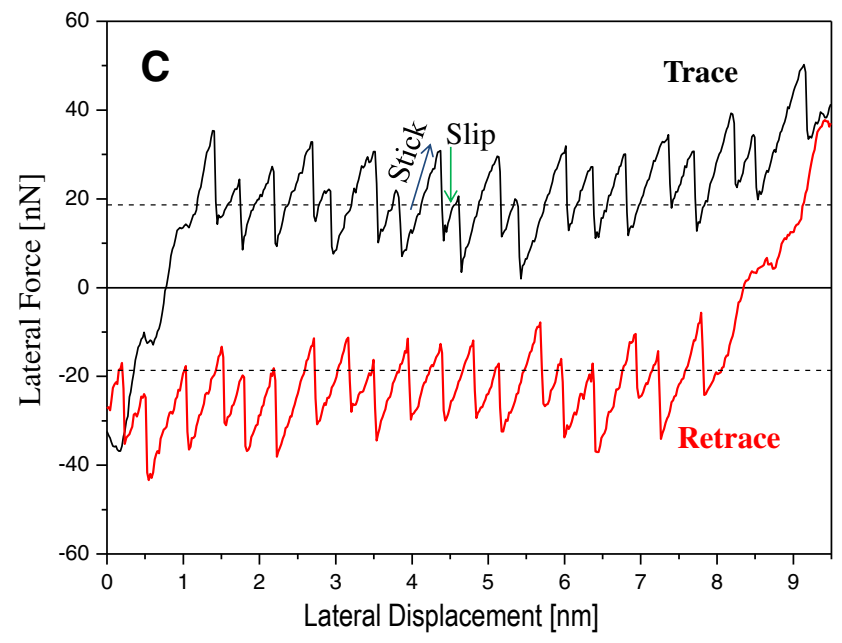

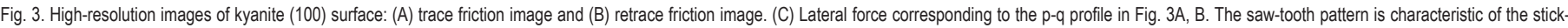
slip phenomenon. Blue arrow shows the stick event and green arrow the slip event. (For interpretation of the references to colour in this figure, the reader is referred to the web version of this article.) 


\section{(i) (ii)}

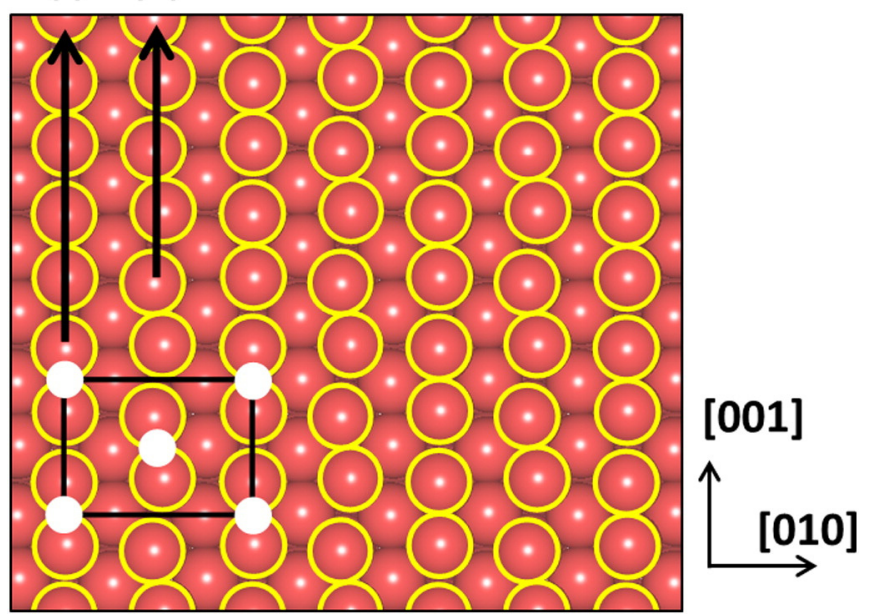

Fig. 4. Kyanite structure projected on its (100) plane. Oxygen is represented by red circles and protruding oxygen are highlighted with yellow rims. Black rectangle shows the sur-face unit cell on the (100) surface. White dots indicate positions where friction maxima presumably occur. The (i) and (ii) black arrows show the two sequences of protruding oxygen (structure projection made with Vesta [15]). (For interpretation of the references to colour in this figure, the reader is referred to the web version of this article.)

contact upon sample rotation. Furthermore, a complete evaluation of the effect of the tip-surface interaction on the image resolution would also require a detailed simulation of tip trajectories based on the Prandtl-Tomlinson model [20], which is beyond the scope of this paper.

\subsection{Load dependence of the friction force}

The average friction forces have been measured on areas of $10 \times 10 \mathrm{~nm}^{2}$ and 500 $\times 500 \mathrm{~nm}^{2}$ while scanning almost parallel to the [010] and [001] directions on this face. Fig. 6 shows the variation of the lateral force, $F_{L}$, with the applied loading (normal) force, $F_{N}$. The applied load ranged from 0 to $40 \mathrm{nN}$. In this range of loading forces, no wear was observed, which is consistent with the relatively high Mohs' hardness of kyanite (100) surface ( $\approx 7$ for the [010] direction, and $\approx 5$ for the [001] direction). The measurements were performed by increasing the normal force and then by decreasing it and using tips with two different nominal radii: SNL-10 D $(2 \mathrm{~nm})$ and NP-10 D $(20 \mathrm{~nm})$. By doing that, we have

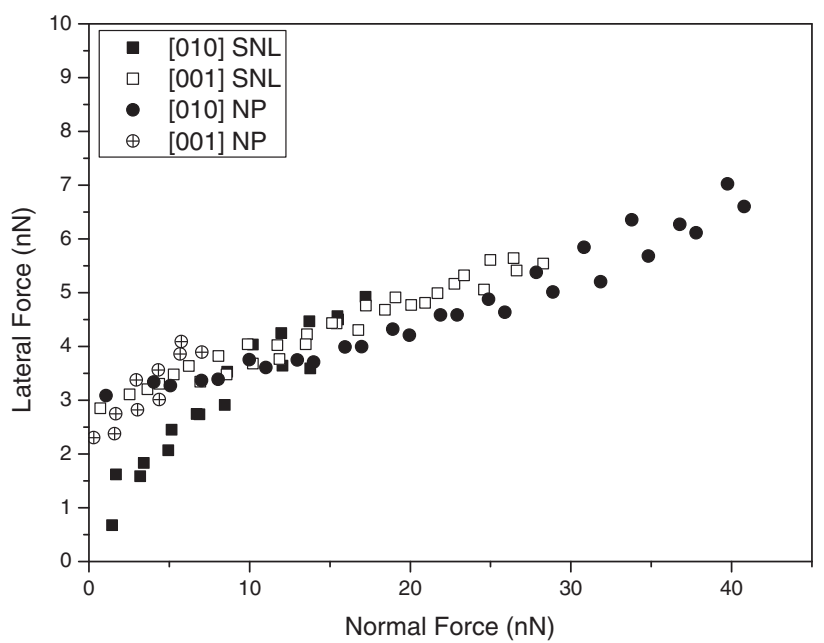

Fig. 6. Lateral force vs. normal force graph measured along directions almost parallel to [010] (solid symbols) and [001] (open symbols). Squares show the experiments performed with SNL-10 D tips and circles show the experiments performed with NP-10 D t i p s .

checked the reproducibility of the measurements and evaluated the effect of tip contact areas on friction. As can be seen in Fig. 6, a s i mil a r d e p e n d e n - cy between loading force and measured friction for the two directions was obtained. Furthermore, the use of tips with different radii did not sig-nificantly affect our friction measurements.

From data shown in Fig. 6, we have calculated the friction coefficient as the slope of the $F_{L}\left(F_{N}\right)$ curves $\left(\mu=d F_{L} / d F_{N}\right)$. The data are almost independent on both the tip radii and the sliding direction, and an aver-age friction coefficient $\mu \approx 0.10$ is obtained in all cases. A discrepancy is observed only in the very first data points acquired with the SNL tip along the [010] direction, where the tip was possibly very sharp and a negligible offset was measured [compared to the value $F_{L}(0) \approx 3 n N$ recorded in the subsequent measurements]. It is quite noticeable that the average values of the friction forces are similar in the two directions despite the different resolution obtained in the FFM maps. While similar friction coefficients indicate comparable tip-surface interactions along the [001] and [010] directions, the different resolution obtained can be related to differences in tip configuration and tip trajectories when the scan is performed along such directions [21].

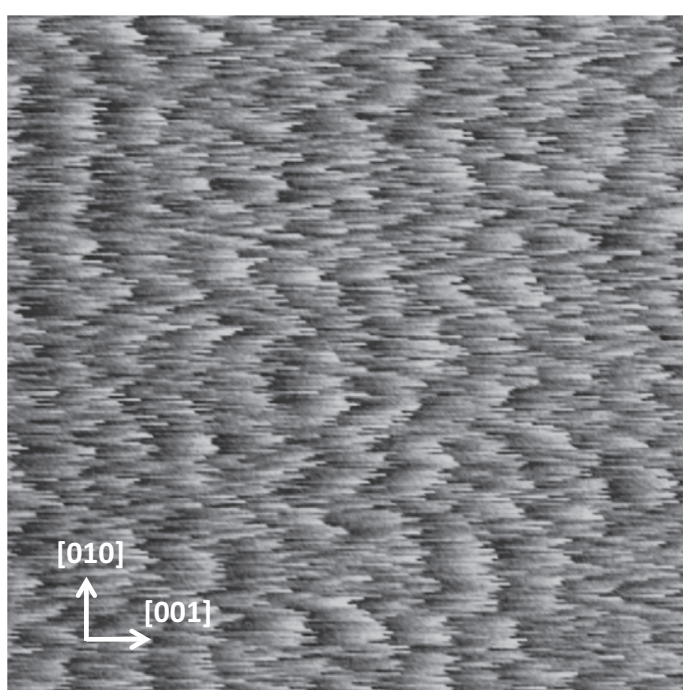

Fig. 5. (A) High-resolution friction image of (100) kyanite surface in water acquired when scanning along [001] direction. Image recorded with $\mathrm{F}_{\mathrm{N}}=23.33 \mathrm{nN}$ and $\mathrm{F}_{\mathrm{L}}=13.40 \mathrm{nN}$. The size of the image is $5.5 \times 5.5 \mathrm{~nm}^{2}$.

\section{Concluding remarks}

1. In this paper, we have reported first images with lattice resolution of the kyanite (100) faces using FFM in water. Maxima in the friction maps reflect the stick-slip motion of the probing tip pulled along the cleavage surface and the convolution between the tip and two protruding oxygen belonging to each $\mathrm{AlO}_{6}$ polyhedron. Interestingly, the resolution of the acquired images varies strongly with the scan direction, indicating different degrees of commensurability between the tip apex and the surface lattice.

2. Maxima in the friction maps define an almost centred rectangular lattice with unit cell dimensions $\left(b_{s}=0.82 \mathrm{~nm} \pm 0.02\right.$ and $\left.c_{s}=0.56 \mathrm{~nm} \pm 0.03\right)$ in good agreement with data derived from the reported bulk structure. Therefore, any significant reconstruction or relaxation phenomena in the kyanite (100) surface structure can be discarded.

\section{Systematic measurements of friction using tips with different radii and} materials (silicon and silicon nitride) provided similar friction coeffi-cients (in the order of $\mu=0.10$ ) when the kyanite (100) surface was scanned along two perpendicular crystallographic directions, i.e., [001] and [010]. 


\section{Conclusions}

Our results can be of interest for theoretical investigations on the relation between surface commensurability and friction anisotropy on the atomic scale, which, so far, have mainly focused on graphite surfaces $[19,20,22]$. An extension of our experimental analysis to different mineral surfaces can be important for understanding the mechanical response of the material under shear with ultimate applications to earthquake dynamics.

\section{Acknowledgements}

This work was supported by the Spanish Ministry of Economy and Competitiveness (Project MAT2012-24487). C.P. acknowledges to Spanish Ministry of Education, Culture and Sports an FPU grant. AFM observations were carried out at ICTS Centro Nacional de Microscopía Electrónica at the Complutense University of Madrid.

\section{References}

[1] P. Puelles, B. Ábalos, J.I. Gil-lbarguchi, Lithos 84 (2005) 125

[2] S. Poli, M.S. Schmidt, Annu. Rev. Earth Planet. Sci. 30 (2002) 207.[3] R.A.

Robie, B.S. Hemingway, Am. Mineral. 69 (1984) 298.
[4] A. Putnis, Introduction to Mineral Sciences, Cambridge University Press, 1992.

[5] A. Tanner, U.S. Geol. Surv. Miner. Commod. Summ. (2014) 88

[6] H. Schneider, A. Majdic, Ceram. Sci. 11 (1981) 191.

[7] A.D. Kustov, O.G. Parfenov, L.A. Solovyov, S.N. Vereshchagin, Int. J. Miner. Process. 126 (2014) 70.

[8] C.M. Pina, R. Miranda, E. Gnecco, Phys. Rev. B 85 (2012) 073402.

[9] C. Pimentel, C.M. Pina, E. Gnecco, Cryst. Growth Des. 13 (2013) 2557.

[10] R. Benages-Vilau, T. Calvet, M.A. Cuevas-Diarte, C. Pimentel, C.M. Pina, Cryst. Growth Des. 13 (2013) 5397.

[11] N. Pawel, C. Pimentel, F. Luo, B. Milian-Medina, J. Gierschner, C.M. Pina, E. Gnecco, Nanoscale 6 (2014) 8334.

[12] C. Pimentel, S. Varghese, S.-J. Yoon, S.Y. Park, J. Gierschner, E. Gnecco, C.M. Pina, J. Mater. Chem. C (2015) (under revision).

[13] P. Comodi, P.F. Zanazzi, S. Poli, M.W. Schmidt, Am. Mineral. 82 (1997) 452.

[14] J.W. Anthony, R.A. Bideaux, K.W. Bladh, M.C. Nichols, Handbook of Mineralogy Mineralogical Society of America1995.

[15] K. Momma, F. Izumi, J. Appl. Crystallogr. 44 (2011) 1272.

[16] R. Lüthi, E. Meyer, H. Haefke, L. Howald, W. Gutmannsbauer, M. Guggisberg, M. Bammerlin, H.J. Güntherodt, Surf. Sci. 338 (1995) 247.

[17] A. Noy, C.D. Frisbie, L.F. Rozsnyai, M.S. Wrighton, C.M. Lieber, J. Am. Chem. Soc. 117 (1995) 7943.

[18] I. Horcas, R. Fernández, J.M. Gómez-Rodríguez, J. Colchero, J. Gómez-Herrero, A.M. Baro, Rev. Sci. Instrum. 78 (2007) 013705.

[19] G.S. Verhoeven, M. Dienwiebel, J.W.M. Frenken, Phys. Rev. B 70 (2004) 165418.[20] E. Gnecco, O.Y. Fajardo, C.M. Pina, J.J. Mazo, Tribol. Lett. 48 (2012) 33

[21] O. Fajardo, E. Gnecco, J.J. Mazo, Phys. B Condens. Matter 455 (2014) 44

[22] S.G. Balakrishna, A.S. de Wijn, R. Bennewitz, Phys. Rev. B 89 (2014) 245440. 